\title{
Relato de Experiência de Monitoria de Análise Experimental do Comportamento: protocolo alternativo de aulas práticas
}

\section{Experience Report of Monitoring on Experimental Analysis of Behavior: Alternative Protocol of Practical Classes. \\ Relato de Experiencia de Monitoreo de Análisis Experimental del Comportamiento: protocolo alternativo de enseñanza de clases prácticas}

\section{Eduardo Sousa Gotti', Elimar Adriana de Oliveira², Djalma Araújo Guimarães Neto³, Cristine Daher Sallum da Cunha ${ }^{4}$, Larissa da Costa Formaji ${ }^{5}$}

Resumo: O presente trabalho tem como objetivo relatar as atividades de monitoria da disciplina de Análise Experimental do Comportamento do curso de Psicologia, da Universidade Federal do Triângulo Mineiro (UFTM), destacando sobre os desafios e implicações da monitoria na construção de repertórios comportamentais para o ensino da Análise Experimental do Comportamento. O programa de monitoria é uma ferramenta adotada por instituições de ensino superior com objetivos diversos, de acordo com cada regulamento de monitoria de cada instituição. A Análise Experimental do Comportamento tem sido vista como uma ramificação da Análise do Comportamento, com pressupostos teóricos no Behaviorismo Radical, filosofia esta que atribui a causalidade do comportamento às interações no ambiente.

Palavras-chave: Palavras-chave: análise experimental do comportamento; ensino; monitoria 
Abstract: The present work aims to report on the monitoring activities on the Experimental Analysis of Behavior subject of the Psychology course at the Federal University of the Triângulo Mineiro (UFTM), highlighting the challenges and implications of monitoring in the construction of behavioral repertoires for the teaching of Experimental Analysis of Behavior. The monitoring program is a tool adopted by higher education institutions with different objectives, according to each institution's monitoring regulations. Experimental Analysis of Behavior has been viewed as a ramification of Behavior Analysis, with theoretical assumptions in Radical Behaviorism, a philosophy that attributes behavioral causality to interactions in the environment.

Keywords: experimental analysis of behavior; teaching; monitoring

Resumen: El presente trabajo tiene como objetivo relatar las actividades de monitoreo de la disciplina de Análisis Experimental del Comportamiento del curso de Psicología, de la Universidad Federal del Triángulo Minero (UFTM), destacando sobre los desafíos e implicaciones del monitoreo en la construcción de repertorios conductuales para la enseñanza de la enseñanza Análisis Experimental del Comportamiento. El programa de monitoreo es una herramienta adoptada por instituciones de enseñanza superior con objetivos diversos, de acuerdo con cada reglamento de monitoreo de cada institución. El Análisis Experimental del Comportamiento ha sido visto como una ramificación del Análisis del Comportamiento, con supuestos teóricos en el Behaviorismo Radical, filosofía que atribuye la causalidad del comportamiento a las interacciones en el ambiente.

Palabras-clave: análisis experimental del comportamiento; enseñanza; monitoreo 


\section{O programa de monitoria nas universidades brasileiras}

A atividade de monitoria foi regulamentada no Brasil em 1968 a partir da Lei Federal n. ${ }^{\circ}$ 5.540, que fixa normas de funcionamento do ensino superior e institui no artigo 41, a monitoria acadêmica, descrevendo que:

\begin{abstract}
"as universidades deverão criar as funções de monitor para alunos do curso de graduação que se submeterem a provas específicas, nas quais demonstrem capacidade de desempenho em atividades técnico-didáticas de determinada disciplina” (BRASIL, 1968).
\end{abstract}

A monitoria durante a graduação está inserida como uma atividade de apoio às disciplinas, de modo geral podendo o aluno monitor experienciar metodologias de ensino, desde que supervisionadas pelo docente da disciplina, além da possibilidade da "solidificação do conhecimento" adquirido ao ter cursado a disciplina à qual se inscreveu como monitor. Essa prática poderá contribuir para a prática didático-pedagógica futura do aluno monitor uma vez que o aproxima das práticas da docência (Matos \& Vivan, 2018).

De modo geral, nas universidades há normas ou estatutos específicos das monitorias, em que há a descrição de critérios de seleção, prazo de inscrição e seleção, atividades que poderão ser desenvolvidas pelo monitor, avaliação e envio de relatórios de atividades de monitoria desenvolvidas às pró-reitorias de ensino. No Estatuto de Monitoria da UFTM, estão previstas atividades, como: auxilio na execução e ou sugestão de materiais ou recursos didáticos, participação de aulas teóricas ou práticas com supervisão do orientador, sugestão nas avaliações, entre outras formas de apoiar a disciplina em suas especificidades. Apoiar, nesse contexto, pode ser entendido, na abordagem da Análise do Comportamento, como uma classe de comportamentos operantes funcionais, que envolvem diferentes tipos (topografias) de comportamentos que devem ser emitidos pelo monitor, como os já mencionados acima. Em síntese, as implicações dessas contribuições e execução de atividades possibilita ao aluno monitor treinar repertórios de ensino, que poderão ser úteis na possível escolha da carreira acadêmica.

Cabe destacar que a monitoria pode beneficiar professor e alunos, visto que o monitor também pode auxiliar na mediação do contato entre professor e aluno durante o processo de ensino e aprendizagem, executando atividades como: receber trabalhos, transmitir informações gerais da disciplina ou esclarecer dúvidas, elaborar orientações de aulas práticas. Isso implica também, na possibilidade de melhora do desempenho em aula pelo aluno e otimização do tempo dos professores uma vez que, estes deparam com realidades extenuantes de um número expressivo de alunos para atender (Gasparinio, Barreto \& Assunção, 2005), e por fim esta mediação pode contribuir para diminuir possíveis obstáculos do ensino e atender os alunos dentro do ritmo dos mesmos (Alves, Souza, Filho \& Ellias. 2011), além de auxiliarem na melhora do aproveitamento da disciplina, que pode ser apreciado a partir do relato dos alunos e das notas por eles obtidas (Matos \& Vivan, 2018).

\section{Ciência do Comportamento e Laboratório Didático: definições e desafios}

A Análise do Comportamento é uma ciência natural que tem por objeto de estudo o comportamento em seus próprios termos, isto é, sem que este seja explicado por meio de construtos mentais ou entidades imateriais (psique, mente, cognição). Trata-se de uma ciência que compreende o comportamento a partir da interação deste com eventos ambientais, e a partir de uma descrição da interação entre esses eventos, objetivando a previsão e controle dos comportamentos (Skinner, 1953/2003). A ciência do comportamento se embasa na filosofia do Behaviorismo Radical, e tem como ramificação da abordagem a Análise Experimental do Comportamento, encarregada da produção e validação de dados empíricos (Neto, 2002).

O método experimental implica na produção de conhecimento empírico que adota planejamentos de manipulações de variáveis em contextos controlados e deliberadamente simplificados e artificias (Neto, 2002). Contudo, é preciso destacar 
que o método experimental não consiste em um método único ou exclusivo para o estudo do comportamento, de maneira que há possibilidades de investigação empírica para além do laboratório que possibilita o estudo das regularidades dos eventos ambientais e comportamentais, tais como observações sistemáticas em ambiente natural e estudos semi-experimentais (Kantowitz, Roediger \& Elmes, 2006; Neto, 2002).

A Análise do Comportamento pode ser tomada como uma forma de lidar com questões que tem sido discutidas pela Psicologia (Hübner \& Moreira, 2012). Diferencia-se das tradicionais explicações de causalidade de comportamento presente em abordagens psicológicas, entendendo o comportamento como variável dependente das variáveis ambientais. Estabelece uma compreensão científica natural sobre os fenômenos comportamentais ao descreve-los a partir de suas interações e dimensões físicas mensuráveis (Moreira \& Medeiros, 2007).

Especificamente dentro do laboratório, há condições que proporcionam ao aluno, conhecer a metodologia experimental, assim como o aprendizado de observação, registro e modificação do comportamento a partir da manipulação dessas variáveis. Outra característica importante do Laboratório de Análise Experimental do Comportamento é a aproximação do aluno de Psicologia da análise de dados quantitativos, no qual a frequência e duração de um comportamento operante é um dado fundamental a ser analisado no laboratório (Matos \& Tomanari, 2002).

No modelo experimental de ensino de análise do comportamento, tradicionalmente vinha sendo utilizado o modelo animal de ratos albinos para a análise de repertórios comportamentais que são importantes na formação profissional do estudante de Psicologia e especificamente do analista do comportamento, tal como: observar, registrar, analisar e manipular variáveis. Além da possibilidade de utilização de ilustração prática de conceitos teóricos básicos da disciplina como: comportamento operante, reforço, punição, extinção, modelagem e esquemas de reforçamento. A despeito das diferenças entre humanos e animais, há princípios que se aplicam aos comportamentos de ambos, no qual o modelo animal pode ser útil para explicar esses princípios, todavia com cautela para que a compa- ração não seja simplista, o que não apenas prejudica a compreensão como também ultrassimplifica a complexidade do comportamento humano (Lopes, Miranda, Nascimento \& Cirino, 2008), dando margens a muitos equívocos e preconceitos em relação à ciência do comportamento.

No que diz respeito aos experimentos que envolvem restrição hídrica em animais, uma das operações estabelecedoras (motivacionais) mais utilizadas em experimentos de processos básicos de aprendizagem operante com animais de laboratório (Tomanari, Pine \& Silva, 2003), paulatinamente a postura do CONCEA ${ }^{1}$ se demonstrou mais acirrada em relação ao período ao quais os organismos vivos ficariam sujeitos à privação. A princípio, o tempo tolerado de acordo com a literatura era de 21 horas (tempo máximo para não produção da desidratação) e recentemente, ficou estabelecido para o contexto de ensino, que tal medida foi inteiramente vedada, sob a seguinte orientação (BRASIL, 2016, p. 7):

\section{“2.2.2.3. Modificação de comportamento ali- mentar ou hídrico \\ Alimento ou líquido pode ser usado como re- compensa, mesmo para animais bem alimenta- dos. Muitas vezes, entretanto, os animais pre- cisam estar com fome ou sede para trabalhar ou realizar uma tarefa para receber alimento ou água como recompensa. Em todos os casos onde o pesquisador possa causar impacto sobre a ingestão de água e alimento, uma justificativa científica, circunstanciada, deve ser fornecida à CEUA, para submeter o protocolo à aprovação. $O$ procedimento não se aplicaria ao uso no en- sino, apenas ao uso científico.}

Assim sendo, de acordo com essa nova normativa, os laboratórios que previam o uso da restrição hídrica para estabelecimento da operação motivadora em seus protocolos de experimentos, estão compelidos a rever as metodologias de aulas práticas no sentido da não utilização de restrição e de

1 As siglas CONCEA e CEUA significam Conselho Nacional de Controle de Experimentação Animal e Comissão de Ética no Uso de Animais 
animais no ensino. Essa mudança nas contingências nas quais trabalham professores, técnicos e alunos induz a variabilidade comportamental destes no contexto das atividades práticas. Em meio a esse contexto, algumas questões de grande relevância são levantadas: existem alternativas didáticas que permitam uma modelagem do comportamento dos estudantes sobre observação e experimentação com comportamentos?

Muitas vezes as críticas ao emprego dos animais à experimentação são dadas da seguinte forma: "É válido submeter os animais a cada semestre letivo a procedimentos já amplamente documentados, sendo que muitas vezes, na sequência das práticas, eles são eutanasiados? Não seria isso um desperdício de vidas e de tempo quando esses dados necessários já foram coletados?" Essa pergunta possível de ser formulada por ativistas em defesa dos direitos dos animais parece se distanciar do objetivo real das práticas de ensino com os animais, uma vez que o objetivo dessas práticas não é a modelagem do comportamento dos animais, mas sim modelagem do comportamento dos alunos para a observação, registro, manipulação de variáveis e análise dos dados obtidos, onde se entende que o modelo animal seria o modelo mais recomendado quando se considera que se pode programar situações mais controladas de interferências no estudo do comportamento, diferentemente do que ocorre em ambientes não-controlados, no qual variáveis incontroláveis poderiam enviesar os dados obtidos (Moreira \& Medeiros, 2007) e do que ocorre com estudos de sujeitos humanos, no qual o seu histórico de interações anterior não pode ser monitorado na maioria das vezes. Ambientes com menor grau de controlabilidade das variáveis e de alta complexidade dos comportamentos, como é o caso de comportamentos humanos, aumentariam as chances de frustrar a aquisição de repertórios mais básicos do estudante de Psicologia durante o estudo da ciência do comportamento (Banaco, 1990).

Embora não consista o escopo do presente trabalho, é importante salientar, que Skinner (1983) pareceu se opor ao exagero de direitos animais quando concebeu essa questão dentro do que conceituou como "libertas nervosa", termo esse que faz analogia à anorexia nervosa, quando alguém engaja em dietas rigorosas para perder pesos, chegando a desnutrição. Skinner (1983), ainda nessa mesma ocasião, aponta para outros problemas de excesso de liberdades individuais que compreendia como sendo um exagero e que poderia colocar em risco a sobrevivência da sociedade, tais como a falta de controle de natalidade, poluição desmedida, consumo de recursos não renováveis e ainda, "direitos de andar de motocicleta sem capacete, direito de usar carros poluentes ou direitos dos fetos". (p. 05). Nesse sentido seria importante que pesquisas futuras discutissem sobre algumas possíveis considerações do debate sobre ética do uso de animais a partir de uma perspectiva analítico comportamental.

Também foi apontada em semestres passados uma alternativa pelo Comitê de Ética com Uso de Animais (CEUA) da UFTM, que sugeriu que os experimentos fossem gravados e que nos semestres posteriores utilizassem essas filmagens. Corroborando para posições como esta, já estão disponíveis livros virtuais que ilustram com vídeos as principais atividades desenvolvidas pelos estudantes de Psicologia nas disciplinas de Análise Experimental do Comportamento, tal como é o caso do livro "Uma história da aprendizagem operante" (Moreira \& Carvalho, 2017). Entretanto, esse tipo de recurso, embora seja ilustrativo sobre as práticas, não permite com que a modelagem do comportamento dos estudantes ocorra e ainda, quando se admite que pode aprender apenas com a observação, está sendo negligenciada a aquisição de habilidades como observação, registro e manipulação de variáveis ambientais e comportamentais, que embora possam ser instruídas por meio de controle de regras (estímulos discriminativos verbais) apenas são fortalecidas no repertório a partir da exposição as contingências de reforço que selecionarão os comportamentos do cientista. Nesse sentido, as vantagens da aprendizagem pela exposição as contingencias diretas sobre a aprendizagem por controle instrucional é tal que possibilita com que o sujeito amplie variações comportamentais e que entrarão em contato com reforçadores naturais, ao passo que as instruções não apenas tenderiam reduzir as variações como seria restrito ao reforço arbitrário de seguir as regras (Matos, 2001).

Muitas universidades no Brasil aderem ao modelo do rato virtual, programa Sniff Pró e, embora a Universidade Federal do Triângulo Mineiro (UFTM) 
conte com software instalado em computadores do Laboratório de Informática, autores renomados da área, defendem a utilização do modelo animal para uma melhor formação do aluno na área de análise experimental (Tomanari \& Eckerman, 2003). Dentre os problemas teóricos presentes no software, comentados pelos autores acima, destacam-se: o ícone "Janela da Mente" que dá margens a noções de causalidade interna (internalismo) do comportamento, que é incompatível com a concepção externalista e selecionista adotada pela Análise do Comportamento; outro problema diz respeito à ausência de emulação de operações motivadoras para o comportamento de pressão a barra; ausência de variabilidade topográfica do comportamento no processo de extinção operante; a emulação do procedimento de modelagem não consiste em aproximações sucessivas a um comportamento alvo por meio de reforço diferencial. Assim, não se pode afirmar que está modelando um operante; no emulador o "condicionamento" é estabelecido a partir de uma acumulação de taxas de associação, o que dá margem a concepções internalistas. Além disso, há definições incorretas no manual da versão 2.0, tais como: "A expressão reforço negativo refere-se ao procedimento de eliminação de um reforço negativo como consequência de um comportamento" (Alloway, Wilson \& Graham, 2006, p. 187).

Todavia, analistas do comportamento também se dividem a respeito da pertinência do emprego do modelo animal nas práticas de ensino de análise experimental do comportamento (Cirino, Miranda, Gonçalves, Miranda, Vieira \& Nascimento, 2010; Lopes, Miranda, Nascimento \& Cirino, 2008), que apontam como um importante argumento o baixo investimento em estudo de comportamento humano comparado a onerosa manutenção de biotérios para comportamentos animais demasiado simples. Contudo, como permite considerar a partir do próprio modelo teórico analítico comportamental, a não utilização do modelo animal não será garantida ou mesmo extinta tão somente pelo recrudescimento das medidas que cerceiam o estudo com os animais, uma vez que elas podem produzir muitos contracontroles que vão desde a variação dos protocolos experimentais para serem aceitos pelos comitês (como uso de água açucarada, achocolatado, uso de dietas com maior taxa de sódio) ou até mesmo estudos clandestinos.
Diante deste cenário, torna-se necessário reforçar positivamente protocolos experimentais com humanos ou alternativos, podendo contrabalancear com a redução na probabilidade dos comportamentos de utilização dos modelos animais. O controle aversivo e toda a pressão exercida por meio das ONGs em defesa dos direitos dos animais, podem consequenciar, a contragosto de seus próprios ideais, mais respostas de contracontrole do que os comportamentos desejáveis. É de se esperar que trabalhando sob condições de controle aversivo, desconsiderando variáveis importantes como a qualidade do ensino ou de baixos reforçadores, tenha efeitos além da qualidade das aulas, como também da motivação para os professores, especialmente quando não há modelos alternativos que equiparem ao ensino de qualidade com a utilização do modelo animal.

Por conta dessas questões colocadas, na disciplina de Análise Experimental do Comportamento do curso de Psicologia da UFTM que até utilizava o modelo animal, no primeiro semestre de 2017, recorreu ao protocolo experimental com humanos, apresentado por Matos e Tomanari (2002) em: A Análise do Comportamento no Laboratório Didático. Segundo Matos e Tomanari (2002), o protocolo experimental consiste na apresentação de 80 verbos em que o sujeito participante escolhido emite uma frase que contenha aquele verbo diante de uma ficha de pronomes, os quais ele pode utilizar de modo aleatório. As primeiras 20 frases que o sujeito participante elaborar serão utilizadas para a construção da linha de base, de maneira que nessa fase os experimentadores se limitam a apenas observar e registrar os comportamentos (frequência de emissão dos pronomes específicos) sem manipular variáveis ambientais consequentes de forma diferencial. A partir da $21^{\circ}$ frase, inicia-se o processo de modelagem para a apresentação de um determinado pronome específico (pronome eu, por exemplo). Neste protocolo, a modelagem é conduzida a partir da apresentação de reforço social (sorriso, elogio, acenar a cabeça com aprovação, parabenização) todas as vezes que o participante emitir frases que contenha o pronome alvo. Durante esse processo, os alunos que aplicam o experimento recebem uma folha para o registro da frequência do comportamento emitido, durante as fases de linha de base e de experimentação. 
No laboratório de Análise Experimental do Comportamento, tanto alunos da disciplina, quanto monitores tiveram participação na aplicação deste protocolo pela primeira vez na disciplina, orientados pelo técnico de nível superior e professora responsável pela disciplina.

\section{Atividades realizadas}

Participaram da monitoria da disciplina de Análise Experimental do Comportamento do curso de Psicologia da UFTM, três monitores que foram devidamente selecionados de acordo com o edital em vigência. Os monitores foram orientados a se dividirem em três turmas, de modo que cada monitor acompanhasse uma turma de alunos da disciplina. As aulas práticas ocorreram uma vez por semana durante duas horas aula, no laboratório de Análise Experimental do Comportamento. Durante as aulas práticas, os participantes, incluindo: monitores, alunos, técnico de nível superior e docente responsável pela disciplina, se dispunham em cadeiras e mesas do laboratório onde eram apresentadas atividades práticas previstas no plano de ensino da disciplina, como: exercícios para auxiliar na compreensão do que havia sido trabalhado durante as aulas teóricas, como exercícios adaptados do livro: "Aprendendo a observar", sobre a importância de observar e registrar os comportamentos de forma clara (não ambígua), simples e objetiva (sem fazer inferências a causalidades internas) (Danna \& Matos, 2011). Além dessas atividades, foi realizado o experimento conforme modelo de Matos e Tomanari (2002), no qual posteriormente os alunos da disciplina elaboraram relatórios dos procedimentos, resultados e discussão dos dados, como uma das formas de avaliação da disciplina.

Durante as primeiras aulas da disciplina, os monitores se encarregaram de elaborar questões sobre as diferenças do Behaviorismo Metodológico e do Behaviorismo Radical que foram utilizadas como roteiro de discussão para os alunos. No decorrer do semestre letivo, os alunos foram orientados pelos monitores quanto à aplicação do experimento de modelagem de comportamento verbal com pronomes. Após orientações, os alunos replicaram esse experimento em três momentos, sendo que o primeiro deles foi realizado no próprio laboratório com supervisão dos monitores, técnico e professora responsável. As duas outras aplicações foram realizadas fora do laboratório em condições arranjadas pelos próprios alunos dentro dos parâmetros previstos pela proposta do experimento. Após as aplicações do experimento de modelagem do comportamento verbal, durante as aulas práticas foram analisados e discutidos os dados coletados, contextualizando com a literatura da área e o conteúdo trabalhado durante as aulas teóricas. Os monitores tiveram participação também nesta fase de análise e discussão dos dados: levantando questões sobre frequência do comportamento, papel de variáveis culturais na construção das frases, orientando a representação dos dados em gráficos.

Durante a análise e discussão dos dados, alguns tópicos interessantes foram levantados pelos alunos, como: a valência do reforço utilizado, que no caso consistia em elogios por parte dos alunos experimentadores. Em um dos grupos, um aluno que foi o sujeito experimental comentou que no dia a dia o colega que fez papel de experimentador é "muito alegre e sorridente" e que quando ele a elogiava durante o experimento ele o fazia "mais sério". Esse dado foi discutido, como a influência da topografia e do contexto sobre a valência do estímulo reforçador, de maneira que, é preciso considerar reforço não como uma propriedade intrínseca, mas como uma função de um estímulo que se define contextualmente, de maneira que como foi notado, que o sorriso "mais contido ou menos espontâneo" na condição experimental não atuava como um reforço social, permitindo discutir a natureza contextual e relacional do fenômeno comportamental.

Alguns alunos que foram sujeitos experimentais relataram descobrir "a lógica" do experimento dizendo que perceberam que determinado pronome era reforçado quando verbalizado. Nesse caso, alguns alunos argumentaram que "seguiram a lógica" do experimento e outros, argumentaram que contracontrolaram, falando frases com outros pronomes. Sobre essa questão, foi comentado que aqueles que "seguiram a lógica do experimento" estavam sobre o controle de regras. Trazer essa discussão, ainda que fugisse do conteúdo programático do semestre, visto que controle por regras é discutido na disciplina Análise do Comportamento, no terceiro período do curso, foi algo necessário, posto 
que muitas explicações dadas a princípio pelos alunos estavam de acordo com princípios mentalistas, tal como "a pessoa fez isso [falou as frases com os pronomes específicos], pois a lógica era que falando esse determinado pronome e que teria a aprovação, o sorriso do experimentador". Nesse sentido, foi especificado sobre o papel do controle das consequências, de modo que se o sorriso e aprovação foi menos reforçador do que as consequências de contracontrolar, as probabilidades das respostas de contracontrole aumentariam. Também foi discutido a baixa frequência da utilização dos pronomes "tu" e "vós" pela maioria dos alunos sujeitos no experimento e o fato desses pronomes não serem comumente utilizados na região sudeste e que isso influencia nos resultados encontrados durante o experimento, no qual quando apareciam eram em pequena frequência. Nesse caso se faz necessário considerar variáveis culturais, tais como apontou Matos e Tomanari (2002) ao discutirem sobre esse tópico do experimento do comportamento verbal. No fim dos experimentos os alunos redigiram um relatório sobre as práticas realizadas na disciplina e contaram com a ajuda dos monitores tanto em relação a teoria e referências bibliográficas, tanto quanto as normas e estrutura de um relatório acadêmico. Uma questão de grande relevância percebida pelos monitores foi o ganho de experiência.

A respeito do que é chamado de ganho de experiência, refere-se aos monitores relatarem ter a oportunidade de discutir os assuntos que envolvem à teoria e a prática diretamente com os alunos sob supervisão, e da mesma forma foram requisitados pelos alunos para orientação nas atividades, o que significa um enriquecimento do repertório do aluno monitor quanto a habilidades relacionadas à docência. Também foi percebido uma maior proximidade dos monitores com o conteúdo da disciplina de Análise Experimental do Comportamento complementando o conhecimento adquirido quando cursaram a disciplina.

Assim, a implementação de novas práticas experimentais induziu variações na forma de ensinar, pois do contrário, a discussão dos dados ficaria subaproveitada. Docente, técnico, alunos e monitores estiveram mais sensíveis às contingências naturais do que às regras, pois do contrário teriam que esperar o próximo semestre letivo para discutir as questões levantadas, como sobre o comportamento de seguir regras e compreender que seguir regras é comportamento mantido por consequências (Skinner, 1991).

O elevado custo de resposta em discutir dados experimentais com humanos nas aulas práticas pode ser um fator que influencia na sua ainda incipiente presença nos espaços das disciplinas de Análise Experimental do Comportamento. A presença de um novo tipo de protocolo experimental implica na revisão de toda a dinâmica tradicional de ensino das aulas práticas de Análise Experimental do Comportamento. Mudar estratégias de ensino de aulas práticas nesta disciplina implica em mudar não apenas as topografias comportamentais de ensino no laboratório, mas uma alteração nas funções que permite novas configurações de ensino na área. Implementar novas práticas permite apontar que o Laboratório de Análise Experimental da UFTM não se restringe a ser um laboratório animal operante (Lopes, Miranda, Nascimento \& Cirino, 2008).

\section{Considerações finais}

O programa de monitoria é uma ferramenta de grande utilidade para o aluno tanto monitor quanto o não monitor ampliando ainda mais o conhecimento do conteúdo da disciplina. Dessa forma, o monitor também passa a conhecer o lado do professor, assim como desenvolver habilidades para possível investimento na carreira acadêmica.A Análise Experimental do Comportamento como uma ramificação da Psicologia tem grande importância na formação do graduando de Psicologia, uma vez que é geralmente o único contato do aluno durante o percurso da graduação com os métodos experimentais das ciências naturais, no qual se busca a explicação do fenômeno comportamental na relação entre o organismo e seu ambiente a partir da descrição de eventos naturais, sem apelos a teses internalistas nas explicações tradicionais de Psicologia. Dessa forma o aluno aprende a observar o comportamento dentro de um ambiente controlado, a registrar, analisar dados, prever e controlar os comportamentos.

Esse trabalho trata de um relato de experiência de monitoria na disciplina de Análise Experimental do Comportamento, do curso de Psicologia da 
UFTM, no período de um semestre letivo. Tal período foi caracterizado pela impossibilidade de trabalhar com animais, o que requereu a aplicação de protocolos experimentais com sujeitos humanos. Esse contexto leva ao questionamento da organização de um laboratório de análise experimental do comportamento, tanto no sentido da necessidade e justificação da utilização de animais para o processo de ensino e aprendizagem da disciplina, quanto no sentido da necessidade e justificação de mais investimento em pesquisa com sujeitos humanos. Quanto a primeira questão, pode ser argumentado que a utilização de animais na disciplina é de grande importância e se justifica pela possibilidade da modelagem do comportamento do aluno de fazer observações e registros acurados do comportamento, habilidade tal que não pode ser aprendida por leitura e teoria. Esse contexto também favoreceu a busca por alternativas como o desenvolvimento de protocolos experimentais com utilização de estímulos reforçadores como água com sacarose em substituição a operação motivadora por privação de água (Guedes, Banaco, Andery, Micheletto, Sério, Gioia, Assis, Benvenuti, 2006). Ainda nessa busca de alternativas, segue a segunda questão, no qual foi percebido durante a experiência de monitoria grande entusiasmo quanto à utilização de protocolo de ensino com humanos, entretanto também foi percebido escassas referências experimentais na literatura nesse sentido, o que leva a possibilidade da utilização do laboratório para o desenvolvimento de delineamentos experimentais para esses fins, investindo em potenciais alternativas promissoras para área.

\section{Referências}

Alloway, T., Wilson, G. \& Graham, J. (2006). Sniffy: o rato virtual. Versão Pro 2.0. São Paulo: Thomson Learning.

Alves, D. T., Souza, S. A. V., Filho, S. C. F. P. \& Elias, W. S. (2011). Análise de metodologia baseada no sistema de ensino individualizado de Keller aplicada em um curso introdutório de eletromagnetismo. Revista Brasileira de Ensino de Física, 33 (1), 1-12.

Banaco, R. A. (1900). O trabalho de laborató- rio na formação em Análise Experimental do Comportamento. Material didático do Laboratório de Psicologia Experimental da Faculdade de Psicologia da Pontifícia Universidade Católica de São Paulo.

BRASIL. Lei no 5.540 de 28 de novembro de 1968. Câmara dos Deputados. Brasília, DF.

BRASIL. Resolução normativa n ${ }^{\circ} 33$, de 18 novembro de 2016. Conselho Nacional de Experimentação. 2.2.2.3. Modificação de comportamento alimentar ou hídrico. Diário Oficial da União, Brasília, DF, n. 222, p. 7, 21 de nov. 2016.

Cirino, S. D., Miranda, R. L., Gonçalves, A. L., Miranda, J. J., Vieira, R. D. \& Nascimento, S. S. (2010). Refletindo sobre o laboratório didático de análise do Comportamento. Perspectivas em Análise do Comportamento, 2 (1) 15-27.

Danna, M. F. \& Matos, M. A. (2011). Aprendendo a observar. São Paulo: EDICON.

Gasparini, S. M., Barreto, S. M. \& Assunção, A. A. (2005). O professor, as condições de trabalho e os efeitos sobre sua saúde. Educação e Pesquisa, 31 (2), 189-199.

Guedes, M. L., Banaco, R. A., Andery, M. A., Micheletto, N. Sério, T. M. A. P., Gioia, P. S., Assis, F. P. \& Benvenuti, M. (2006). O estudo de processos comportamentais básicos no laboratório. Laboratório de Psicologia Experimental Programa de Estudos Pósgraduados em Psicologia Experimental: Análise do Comportamento. Disponível em: http:// www.pucsp.br/pos-graduacao/mestrado-e-doutorado/psicologia-experimental-analise-do-comportamento\#publicacoes. Acessado em 04 de Dezembro de 2017.

Hübner, M. M. C. \& Moreira, M. B. (2012). Fundamentos de Psicologia: Temas clássicos de psicologia sob a ótica da Análise do Comportamento. Porto Alegre: Guanabara Koogan.

Kantowitz, B. H., Roediger, H. L \&Elmes, D. G. (2006). Psicologia experimental. Psicologia para compreender a pesquisa em psicologia. São Paulo: Cengage.

Lopes, M. G., Miranda, R. L., Nascimento, S. S. \& Cirino, S. D. (2008). Discutindo o uso do laboratório de análise do comportamento no ensino de psicologia. Revista Brasileira de Terapia 
Comportamental e Cognitiva, 10 (1) 67-79.

Matos, M. A. (2001). Comportamento governado por regras. Revista Brasileira de Terapia Comportamental e Cognitiva, 3 (2) 51-66.

Matos, M. A. \& Tomanari, G. Y. (2002). A análise do comportamento no laboratório didático. Barueri - SP: Editora Manole.

Matos, C. M. V. \& Vivan, A. M. (2018). Relato de experiência de monitoria em uma disciplina de análise experimental do comportamento. Perspectivas em Análise do Comportamento, 9 (1), $80-89$.

Moreira, M. B. \& Carvalho, L. C. (2017). Uma história da aprendizagem operante. Instituto Walden4. Brasília, Distrito Federal.

Moreira, M. B. \& Medeiros, C. A. (2007). Princípios básicos de análise do comportamento. Porto Alegre: Artmed.

Neto, M. B. C. (2002). Análise do comportamento: behaviorismo radical, análise experimental do comportamento e análise aplicada do comportamento. Interação em Psicologia, 6 (1), 13-18.

Skinner, B. F. (1983). Estado de alerta máximo; o grande papa da Ciência do Comportamento identifica em problemas como ameaça nuclear ou a superpopulação perigos inéditos para o mundo. Veja, v. 771, 03-06.

Skinner, B. F. (1991). Questões recentes na análise comportamental. Campinas: Papirus. (Original publicado em 1989).

Skinner, B. F. (2003). Ciência e comportamento humano. São Paulo: Martins Fontes. (Original publicado em 1953).

Tomanari, G. Y. \& Eckerman, D. A. (2003). O rato Sniffy vai à escola. Psicologia: Teoria e Pesquisa, 19 (2) 159-164.

Tomanari, G. Y., Pine, A. S. \& Silva, M. T. A. (2003). Ratos Wistar sob regimes rotineiros de restrição hídrica e alimentar. Revista Brasileira de Terapia Comportamental e Cognitiva, 5 (1) 57-71.
Informações do Artigo

Histórico do artigo:

Submetido em: 03/01/2018

Aceito em: 26/06/2019 